\title{
L'HOMME L'Homme
}

$180 \mid 2006$

Rendre visible

\section{Laurence Alfonsi, Le Cinéma du futur : les enjeux des nouvelles technologies de l'image}

Paris, L'Harmattan/Saint-Nicolas, Les Presses de l'université de Laval 2005, 86 p., bibl. (« Cinéma et Société »).

Jean-Paul Colleyn

\section{(e) OpenEdition}

Journals

Édition électronique

URL : http://journals.openedition.org//homme/2569

DOI : $10.4000 /$ /homme.2569

ISSN : 1953-8103

Éditeur

Éditions de l'EHESS

Édition imprimée

Date de publication : 1 décembre 2006

Pagination : 209-211

ISSN : 0439-4216

Référence électronique

Jean-Paul Colleyn, «Laurence Alfonsi, Le Cinéma du futur : les enjeux des nouvelles technologies de l'image », L'Homme [En ligne], 180 | 2006, mis en ligne le 25 octobre 2006, consulté le 25 septembre 2020. URL : http://journals.openedition.org//homme/2569; DOI : https://doi.org/10.4000//homme. 2569

Ce document a été généré automatiquement le 25 septembre 2020

(c) École des hautes études en sciences sociales 


\section{Laurence Alfonsi, Le Cinéma du futur : les enjeux des nouvelles technologies de l'image}

Paris, L'Harmattan/Saint-Nicolas, Les Presses de l'université de Laval 2005, 86 p., bibl. («Cinéma et Société »).

Jean-Paul Colleyn

LAURENCE ALFONSI retrace l'histoire des techniques cinématographiques et analyse les «futurs possibles » liés au virtuel, à internet, au cinéma domestique (home cinema), à l'interactivité, aux DVD, au cinéma multiplex, au cinéma total, aux images en trois dimensions, etc. Elle part du point de vue que le cinéma n'évolue pas tout seul, mais en synergie avec l'ensemble des "médiasphères"; ce concept désignant un milieu technique axé sur un média dominant et qui engendre un certain rapport à l'espace et au temps. Le virtuel n'est pas seulement aujourd'hui au service d'un imaginaire fantasmatique, il intervient aussi dans le travail de toutes les images et de tous les sons - pour retoucher, modifier, insérer, " améliorer ", truquer, etc. - de telle sorte que la distinction entre image de synthèse et image «réelle» devient pratiquement indiscernable. De nombreux auteurs estiment que le virtuel menace à terme l'existence même du cinéma. Or, d'après Laurence Alfonsi, l'hypothèse d'un tout virtuel est peu crédible. Sans être un enjeu vital, le virtuel serait plutôt une "révolution secondaire " décisive. Ces techniques changent en effet irrémédiablement le statut de l'image, puisqu'on peut facilement mélanger les scènes virtuelles ou des éléments virtuels à des tournages in situ avec des acteurs de chair et d'os. Grâce au digital composing et au morphing, un réalisateur peut faire intervenir un acteur dans des scènes d'archives, prêter des propos qu'il n'a jamais tenus à un personnage historique, ou redonner vie à un acteur prématurément disparu. «La perspective d'une réalité mixte ou augmentée, qui supprime les frontières entre monde tangible et virtuel ", écrit Alfonsi, «oblige donc le critique à craindre une révolution des principes phénoménologiques du cinéma » (p. 12). 
Quel sera, par ailleurs, l'impact des jeux vidéo sur le cinéma? Les deux médiums demeurent assez hétérogènes; en revanche, depuis qu'internet est devenu un vecteur de diffusion de films, il suscite une esthétique nouvelle, fondée sur la vitesse, le caractère éphémère et sur une plus grande sensibilité à l'influence des jeux vidéo. Le cinéma domestique, le ciné cocooning, rendu possible par une haute technologie à domicile (grand écran, enceintes sonores géantes, interactivité), contribue à désacraliser le cinéma et, en quelque sorte, à le privatiser. Mais l'interactivité sera surtout mise en œuvre par les DVD, qui peuvent comporter de véritables ateliers de montage, permettant au spectateur de remonter le film à sa guise, de choisir d'autres fins, d'envisager des récits parallèles, etc. Tout porte à penser néanmoins que le cinéma en salle (fondamentalement rétif à l'interactivité) gardera son charme et une spécificité fondée sur la «sortie en ville» et sur le choix d'une histoire imposée. Tout comme Laurence Alfonsi, je ne crois pas que ce soit l'aspect cérémoniel, ni la volonté d'être ensemble qui garantissent la suprématie du cinéma. De nombreux spécialistes exagèrent, en effet, l'aspect communiel de la salle de cinéma où, sauf exception, le spectateur se trouve tout de même face au film, dans la posture du " chacun pour soi ». En revanche, la qualité supérieure de l'image et du son dans les salles de cinéma restera sans doute pour longtemps un critère décisif et l'auteur a raison d'affirmer que, face à la concurrence du cinéma domestique, le septième art est sommé de développer une véritable mise en scène de la réception. Le mythe du cinéma total refait donc surface. Un mythe qui renferme une certaine ambiguïté entre l'idée d'une représentation parfaite de la réalité et celle d'une illusion de réalisme parfaite, ce qui n'est pas du tout la même chose. Quoi qu'il en soit, les expériences actuelles s'efforçant de rapprocher les perfectionnements techniques des capacités sensorielles humaines n'ont pas fini de nous surprendre.

3 Je passe sur des points évoqués par l'auteur, tels que la tendance hégémonique de Hollywood (qui s'explique non seulement parce que ce cinéma s'exporte partout, mais aussi parce qu'il s'imite partout), la tension art/commerce, le rapport étroit du film avec la violence (déjà soulevé par Walter Benjamin) et bien d'autres questions encore. Le véritable sujet du livre porte en effet sur une " prospective du cinéma », sur l'art de découvrir les futurs possibles, les "futuribles». L'auteur fait ici explicitement référence au groupe et à la revue Futuribles, dirigés par Hugues de Jouvenel. Il est à mettre à son crédit de ne pas vouloir jouer les prophètes de malheur, comme c'est si souvent le cas à propos d'un art dont les amoureux les plus fidèles proclament inlassablement la mort. Son livre est fort utile, car il dessine les évolutions possibles pour le second siècle du cinéma, même s'il ne mène pas la démarche prospective jusqu'au bout. En effet, bien que cette école de pensée se fonde en grande partie sur la théorie des scénarios, Laurence Alfonsi ne l'applique pas systématiquement au cinéma (qui est précisément l'art de mettre en scène des scénarios). On serait curieux de connaître les travaux des prospectivistes de l'industrie hollywoodienne, qui sont malheureusement rarement publiés. La démarche des scénarios pourrait s'avérer féconde au-delà de l'esquisse, mais ce serait un chantier de très grande ampleur car on peut voir le cinéma comme un "fait social total», avec des aspects économiques, sociaux, esthétiques, psychanalytiques et même, pourrait-on dire, religieux puisque le cinéma a fondamentalement à voir avec la croyance ${ }^{1}$.

Bref, parlons du livre que nous sommes chargés de commenter et non d'un autre encore à écrire: Le Cinéma du futur de Laurence Alfonsi apporte de très précieuses 
précisions à une histoire du cinéma en rapport avec les progrès technologiques. On peut regretter que l'auteur ne propose pas de véritable périodisation (ou ne s'explique pas sur l'éventuelle impossibilité de le faire). Des formules comme «Mais il faudra attendre 1980, pour...", "Mais le véritable tournant eut lieu en...", "Un premier tournant s'était opéré... ", " Mais ce n'est qu'au milieu des années 1990, que... », sans doute employées par commodité narrative, présentent le désavantage de suggérer une vision téléologique de l'histoire des techniques; ce qui ne me paraît pas correspondre aux intentions de l'auteur. En revanche, le livre a le mérite de corriger un certain dédain de la critique pour la technique et de souligner l'aspect véritablement passionnel de la vie des inventeurs. Le lecteur relèvera au passage quelques belles phrases sur 2001, l'Odyssée de l'espace de Stanley Kubrick (1968), un film qui, outre son intérêt "philosophique", a totalement renouvelé le rapport du cinéma à la technologie.

Les vertus didactiques du livre rendront certainement service aux étudiants. La bibliographie, copieuse, révèle un souci louable de pédagogie. En effet, il s'agit (à part quelques classiques traduits) d'ouvrages accessibles, écrits en français et pas nécessairement cités dans le texte. Laurence Alfonsi résume, sans trop prendre parti, les positions de spécialistes des médias comme Dominique Wolton et Nicholas Negroponte, de philosophes comme Jean Baudrillard et Paul Virillo, de sociologues comme Michel Maffesoli, etc. Elle pratique une neutralité axiologique qui a ses avantages et ses inconvénients. Ses avantages, car Laurence Alfonsi a l'art de synthétiser et d'exposer clairement la thèse d'un auteur ; ses inconvénients parce que, parfois, un débat majeur se trouve relégué en note, comme la question de savoir si les goûts du public peuvent être, en dernière analyse, façonnés par un diktat économique (p. 31). La conclusion du livre est intéressante, car elle relie l'évolution des techniques à celle de la société. Ce ne seraient pas tant l'incroyable vitesse des changements techniques, les guerres commerciales, la diversification des médias, l'accès du grand public à des techniques sophistiquées, que l'éclectisme philosophique, l'incertitude des valeurs, la dissolution d'une normativité générale, l'idéologie de l'urgence perpétuelle, qui marqueraient le deuxième siècle du cinéma. Laurence Alfonsi termine son livre comme elle l'a commencé, par la métaphore du Titanic, dont un photogramme illustre la couverture. Une image qui symboliserait une société à la fois présomptueuse, fragile et ostentatoire.

\section{NOTES}

1.. Voir Christian Metz, Le Signifiant imaginaire : psychanalyse et cinéma, Paris, Union générale d'éditions, 1977. 